\title{
High precision ${ }^{113} \operatorname{In}(\alpha, \alpha){ }^{113}$ In elastic scattering at energies near the Coulomb barrier for the astrophysical $\gamma$ process
}

\author{
G. G. Kiss, ${ }^{1, *}$ P. Mohr, ${ }^{1,2}$ Zs. Fülöp, ${ }^{1}$ T. Rauscher ${ }^{1,3,4}$ Gy. Gyürky, ${ }^{1}$ T. Szücs, ${ }^{1}$ Z. Halász, ${ }^{1,5}$ E. Somorjai, ${ }^{1}$ \\ A. Ornelas, ${ }^{1,6}$ C. Yalçın, ${ }^{7}$ R. T. Güray, ${ }^{7}$ and N. Özkan ${ }^{7}$ \\ ${ }^{1}$ Institute for Nuclear Research (MTA ATOMKI), P.O. Box 51, H-4001 Debrecen, Hungary \\ ${ }^{2}$ Diakonie-Klinikum, D-74523 Schwäbisch Hall, Germany \\ ${ }^{3}$ Centre for Astrophysics Research, School of Physics, Astronomy and Mathematics, \\ University of Hertfordshire, Hatfield AL10 9AB, United Kingdom \\ ${ }^{4}$ Department of Physics, University of Basel, CH-4056 Basel, Switzerland \\ ${ }^{5}$ University of Debrecen, Department of Theoretical Physics, H-4001 Debrecen, Hungary \\ ${ }^{6}$ Centro de Física Nuclear da Universidade de Lisboa, 1649-003 Lisbon, Portugal \\ ${ }^{7}$ Kocaeli University, Department of Physics, TR-41380 Umuttepe, Kocaeli, Turkey \\ (Received 24 April 2013; revised manuscript received 25 June 2013; published 28 October 2013)
}

\begin{abstract}
Background: The $\gamma$ process in supernova explosions is thought to explain the origin of proton-rich isotopes between $\mathrm{Se}$ and $\mathrm{Hg}$, the so-called $p$ nuclei. The majority of the reaction rates for $\gamma$ process reaction network studies have to be predicted in Hauser-Feshbach statistical model calculations using global optical potential parametrizations. While the nucleon + nucleus optical potential is fairly well known, for the $\alpha+$ nucleus optical potential several different parametrizations exist and large deviations are found between the predictions calculated using different parameter sets.

Purpose: By the measurement of elastic $\alpha$-scattering angular distributions at energies around the Coulomb barrier a comprehensive test for the different global $\alpha+$ nucleus optical potential parameter sets is provided.

Methods: Between $20^{\circ}$ and $175^{\circ}$ complete elastic alpha scattering angular distributions were measured on the ${ }^{113}$ In $p$ nucleus with high precision at $E_{\text {c.m. }}=15.59$ and $18.82 \mathrm{MeV}$.

Results: The elastic scattering cross sections of the ${ }^{113} \operatorname{In}(\alpha, \alpha){ }^{113} \mathrm{In}$ reaction were measured for the first time at energies close to the astrophysically relevant energy region. The high precision experimental data were used to evaluate the predictions of the recent global and regional $\alpha+$ nucleus optical potentials. Parameters for a local $\alpha+$ nucleus optical potential were derived from the measured angular distributions.

Conclusions: Predictions for the reaction cross sections of ${ }^{113} \operatorname{In}(\alpha, \gamma)^{117} \mathrm{Sb}$ and ${ }^{113} \operatorname{In}(\alpha, \mathrm{n}){ }^{116} \mathrm{Sb}$ at astrophysically relevant energies were given using the global and local optical potential parametrizations.
\end{abstract}

DOI: 10.1103/PhysRevC.88.045804

PACS number(s): 24.10.Ht, 25.55.Ci

\section{INTRODUCTION}

Studies in the fields of nuclear structure, nuclear reaction theory, and nuclear astrophysics require the knowledge of $\alpha+$ nucleus optical model potentials (OMPs). For example, the OMP plays a role in the determination of the $\alpha$-decay half-lives of superheavy nuclei [1,2], and in the unification of the bound and scattering $\alpha$-particle states [3]. Furthermore, in several astrophysical applications-such as modeling the nucleosynthesis in explosive scenarios like the $\gamma$ process - the reaction rates are taken from the Hauser-Feshbach $(\mathrm{H}-\mathrm{F})$ statistical model [4] using global OMPs [5,6]. Considerable efforts have been devoted in recent years to improve the $\alpha+$ nucleus optical potential parametrizations for astrophysical applications [7-9]. In the present work, a comprehensive experimental test of the most recent global OMPs used in $\gamma$ process network simulations is carried out for the target nucleus ${ }^{113} \mathrm{In}$, which is traditionally considered a so-called $p$ nucleus [10-12]. Typically, ${ }^{113} \mathrm{In}$ is underproduced in nucleosynthesis calculations of the $p$ or $\gamma$ process. Surprisingly, this

\footnotetext{
*ggkiss@atomki.mta.hu
}

underproduction has not attracted much attention although no alternative production mechanisms have been clearly identified yet [12-15].

\section{A. The astrophysical $\gamma$ process}

About 99\% of the isotopes heavier than iron are synthesized via neutron capture reactions in the so-called $s$ and $r$ processes [16]. However, on the proton-rich side of the valley of stability there are about 35 nuclei separated from the path of the neutron capture processes. These mostly even-even isotopes between ${ }^{74} \mathrm{Se}$ and ${ }^{196} \mathrm{Hg}$ are the so-called $p$ nuclei [16]. It is generally accepted that the main stellar mechanism synthesizing the $p$ nuclei-the so-called $\gamma$ process-involves mainly photodisintegrations, dominantly $(\gamma, n)$ reactions on preexisting more neutron-rich $s$ and $r$ seed nuclei. The high energy photons-necessary for the $\gamma$-induced reactionsare available in explosive nucleosynthetic scenarios where temperatures around a few GK are reached, like the Ne/O rich layer in core-collapse supernovae $[10,18]$ or during the thermonuclear explosion of a white dwarf (type Ia supernova) [17]. Regardless of the astrophysical site, consecutive $(\gamma, n)$ 
reactions drive the material towards the proton rich side of the valley of stability. As the neutron separation energy increases along this path, $(\gamma, p)$ and $(\gamma, \alpha)$ reactions become faster and process the material towards lighter elements [12,19,20]. Theoretical investigations agree that $(\gamma, p)$ reactions are more important for the lighter $p$ nuclei, whereas $(\gamma, \alpha)$ reactions are mainly important at higher masses (neutron number $N \geqslant 82$ ) [16].

Modeling the synthesis of the $p$ nuclei and calculating their abundances requires an extended reaction network calculation involving more than $10^{4}$ reactions on about 2000 mostly unstable nuclei. The necessary cross sections are calculated using the H-F statistical model [4] which utilizes global OMPs. Since the calculated $p$ abundances are very sensitive to the applied reaction rates $[19,20]$ — which are derived by folding the reaction cross sections under stellar conditions with the Maxwell-Boltzmann distribution at a given temperatureexperimental verification of the calculated cross sections is very important. For photodisintegration reactions with charged particle emission there is only a very limited number of cases in the relevant mass and energy range where the $\mathrm{H}-\mathrm{F}$ cross sections can be directly compared to experimental data [21]. Consequently, the model calculations remain mainly untested. However, by using the detailed balance theorem, information on the photodisintegration cross sections can be obtained from the experimental study of the inverse capture reactions. This approach provides more relevant astrophysical information than the direct study of the $\gamma$-induced reactions since often the influence of thermally excited states is smaller in this direction, compared to photon-induced reactions [16,22-24]. In recent years several $\alpha$-capture cross sections have been measured using the well known activation technique [25-33], and the results were compared with the H-F predictions. In general, it was found that the H-F cross sections are very sensitive to the choice of the $\alpha+$ nucleus OMP, in particular at energies significantly below the Coulomb barrier, which is the most relevant energy range for the calculation of stellar reaction rates.

\section{B. Optical potential parametrization}

The optical potential combines a Coulomb term with the complex form of the nuclear potential, which consists of a real and an imaginary part. Usually, the parameters of the OMP are derived from the analysis of the angular distributions of elastically scattered $\alpha$ particles (and are adjusted to experimental $\alpha$-induced cross sections if they are known).

The variation of the potential parameters of the real part as a function of mass and energy is smooth and relatively well understood [34]. On the contrary, the imaginary part of the optical potential is strongly energy-dependent especially at energies around the Coulomb barrier. In astrophysical applications the parameters of the OMP have to be known at energies well below the Coulomb barrier. However, at such energies the $\alpha+$ nucleus elastic scattering cross section is nondiffractive and dominated by the Rutherford component. Therefore, the elastic $\alpha$ scattering experiments have to be carried out at slightly higher energies with high precision.
From the analysis of the measured angular distributions the parameters of the potential can be derived and have to be extrapolated down to the astrophysically relevant energy region where the relevant $\alpha$-particle induced-reactions are taking place.

Several $\alpha$ elastic scattering experiments on the target nuclei ${ }^{89} \mathrm{Y},{ }^{92} \mathrm{Mo},{ }^{106,110,116} \mathrm{Cd},{ }^{112,124} \mathrm{Sn}$, and ${ }^{144} \mathrm{Sm}$ have been performed at ATOMKI in recent years [35-40]. A summary of this work in given in $[9,41]$. In most cases either semimagic or even-even target nuclei were investigated. This work presents the elastic scattering experiment performed on the ${ }^{113}$ In nucleus to study further the behavior of the optical potentials at low energies. In all of these cases complete angular distributions have been measured at energies close to the Coulomb barrier. The chosen energies were low enough to be close to the region of astrophysical interest and high enough that the scattering cross section differs sufficiently from the Rutherford cross section.

The first studies have focused on semimagic even-even nuclei with $N=82\left({ }^{144} \mathrm{Sm}\right), N=50\left({ }^{92} \mathrm{Mo}\right)$, and $Z=50$ $\left({ }^{112,124} \mathrm{Sn}\right)$. These works were extended to investigate the variation of the parameters of the OMP along the $N=50$ and $Z=48$ isotonic and isotopic chains by the study of the ${ }^{89} \mathrm{Y}(\alpha, \alpha)$ and ${ }^{106,110,116} \mathrm{Cd}(\alpha, \alpha)$ reactions [39,40]. Based on the high precision data measured at ATOMKI, a new global OMP has been developed [9]. This few-parameter OMP gives a correct description for the total $\alpha$-induced cross sections [41] and reasonable prediction for $\alpha$ elastic scattering angular distributions. Further $\alpha$ elastic scattering angular distributions at low energies along the Te isotopic chain have been measured at the University of Notre Dame recently [7], and a regional OMP has been fitted to their data. Thus, besides the astrophysical motivation the main aim for the present experiment is to provide an independent check for the recent OMPs for the nonmagic $p$ nucleus ${ }^{113} \mathrm{In}$.

Angular distributions have been measured at $E_{\text {c.m. }}=15.59$ and $18.82 \mathrm{MeV}$, just above and below the Coulomb barrier (the height of the Coulomb barrier for the ${ }^{113} \mathrm{In}+\alpha$ system is about $16 \mathrm{MeV}$ ). At these energies a reliable test for the global parametrization is possible using the new high precision angular distributions. Furthermore, the available $\alpha$-induced cross section data, taken from literature [30], are used to test the $\mathrm{H}-\mathrm{F}$ predictions for the cross sections of the ${ }^{113} \operatorname{In}(\alpha, \gamma){ }^{117} \mathrm{Sb}$ and ${ }^{113} \operatorname{In}(\alpha, \mathrm{n}){ }^{116} \mathrm{Sb}$ reactions, calculated using the recent global/regional OMPs.

\section{EXPERIMENTAL TECHNIQUE}

The experiment was carried out at the cyclotron laboratory of ATOMKI, Debrecen. A similar experimental setup was used in previous experiments [35-40] and is described in more detail in [42]. The following paragraphs provide a short description of the experimental setup.

\section{A. Target production and beam properties}

The targets were produced by evaporation of metallic, highly enriched $(93.1 \%){ }^{113}$ In onto thin carbon foil 
( $\approx 40 \mu \mathrm{g} / \mathrm{cm}^{2}$ ). The thickness was determined by measuring the energy loss of alpha particles emitted by an ${ }^{241}$ Am source using an ORTEC SOLOIST $\alpha$-spectrometer [43]. The target thickness was found to be $142 \mu \mathrm{g} / \mathrm{cm}^{2}$ with an uncertainty of $9 \%$; this corresponds to about $7.6 \times 10^{17}$ atoms $/ \mathrm{cm}^{2}$. For the angular calibration (see below) similar carbon foils to the ones used as backing were applied. The ${ }^{113}$ In and carbon targets, together with the two collimators used for beam tuning, were mounted on a remotely controlled target ladder in the center of the scattering chamber.

The energy of the alpha beam was $E_{\text {lab }}=16.15$ and $19.50 \mathrm{MeV}$, with a beam current of $150 \mathrm{pnA}$. At first a collimator of $6 \times 6 \mathrm{~mm}^{2}$, then a collimator of $2 \times 6 \mathrm{~mm}^{2}$ was used for focusing. We optimized the beam until not more than $1 \%$ of the total beam current could be measured on the smaller aperture. As a result of the procedure, the horizontal size of the beamspot was below $2 \mathrm{~mm}$ during the whole experiment, which is crucial for the precise determination of the scattering angle. Furthermore, the collimators were used also to check the beam position and size of the beamspot before and after every change of the beam energy or current. Since the imaginary part of the optical potential depends sensitively on the energy, it is important to have a well defined beam energy. Therefore the beam was collimated by tight slits ( $1 \mathrm{~mm}$ wide $)$ after the analyzing magnet; this corresponds to an overall energy spread of around $100 \mathrm{keV}$ which is the dominating contribution to the energy resolution of the spectra.

\section{B. Detectors and angular calibration}

Altogether seven ion implanted silicon detectors with active areas of $50 \mathrm{~mm}^{2}$ and $500 \mu \mathrm{m}$ thickness were used for the measurement of the angular distributions. The detectors were collimated with about $1 \mathrm{~mm}$ wide slits and were mounted on two turntables. Two detectors with angular separation of $10^{\circ}$ were mounted on the upper turntable, these detectors were used to measure the yield of the scattered alpha particles at forward angles. Five additional detectors were placed on the lower turntable, in this case the angular separation between the detectors was $5^{\circ}$. The solid angles were typically within $\Delta \Omega=1.0 \times 10^{-4}$ sr and $\Delta \Omega=1.6 \times 10^{-4}$ sr. The ratios of solid angles of the different detectors were checked by measurements at overlapping angles with good statistics.

In addition, two detectors were mounted at a larger distance on the wall of the scattering chamber at fixed angles $\vartheta= \pm 15^{\circ}$ left and right of the beam axis. These detectors were used as monitor detectors during the experiment to normalize the measured angular distribution and to determine the precise position of the beam on the target. The solid angle of these detectors was $\Delta \Omega=8.2 \times 10^{-6} \mathrm{sr}$.

The energy of the first excited state of the ${ }^{113}$ In nucleus is $339.7 \mathrm{keV}$ [44]. There is a large difference between the spin of the ground and the first excited states $\left(9 / 2^{+}\right.$and $1 / 2^{-}$ respectively). Therefore the expected inelastic scattering cross section leading to this excited state is very low (below $0.44 \mathrm{mb}$, calculated with the TALYS code [45]) at the measured energies. (a)
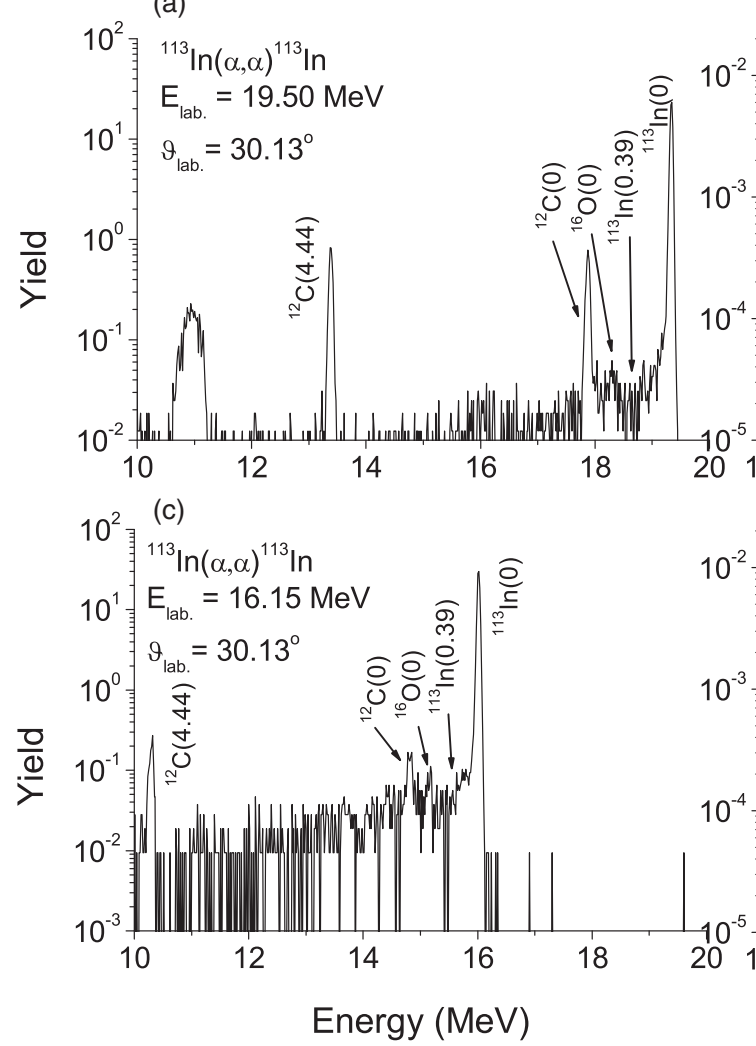

(b)

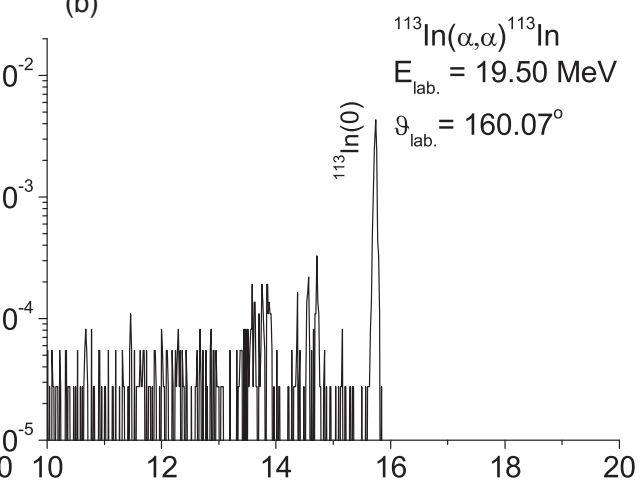

(d)

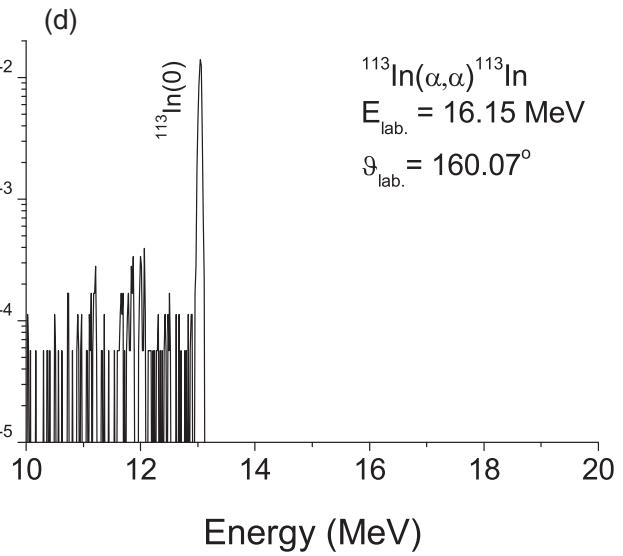

FIG. 1. Typical spectra at $E_{\text {lab }}=19.50 \mathrm{MeV}[(\mathrm{a})$ and (b) $]$ and $16.15 \mathrm{MeV}\left[(\mathrm{c})\right.$ and (d)], measured at $\vartheta_{\text {lab }}=30.13^{\circ}[(\mathrm{a})$ and (c) $]$ and $160.07^{\circ}$ [(b) and (d)]. The peak from elastic ${ }^{113} \mathrm{In}+\alpha$ scattering is well resolved from both the ${ }^{12} \mathrm{C}+\alpha$ and ${ }^{16} \mathrm{O}+\alpha$ elastic scattering. 
Typical spectra are shown in Fig. 1. The relevant peaks from elastic ${ }^{113} \mathrm{In}+\alpha$ scattering are well separated from elastic and inelastic peaks of target contaminations, and-as expectedpeaks from inelastic $\alpha$ scattering on ${ }^{113}$ In are practically not visible.

Knowledge of the exact angular position of the detectors is of crucial importance for the precision of a scattering experiment since the Rutherford cross section depends sensitively on the angle. The uncertainty of the cross section at forward angles in the angular distribution is dominated by the error of the scattering angles. A tiny uncertainty of $\Delta \vartheta=0.3^{\circ}$ results in a significant error of approximately $5 \%$ in the Rutherford normalized cross sections at very forward angles.

To determine the scattering angle precisely, we measured kinematic coincidences between elastically scattered $\alpha$ particles and the corresponding ${ }^{12} \mathrm{C}$ recoil nuclei at $E_{\text {lab }}=$ 16.15 MeV, using a pure carbon foil target. One detector was placed at $\vartheta=60^{\circ}$ and the signals from the elastically scattered $\alpha$ particles on ${ }^{12} \mathrm{C}$ were selected as gates for the other detector, which moved around the expected ${ }^{12} \mathrm{C}$ recoil angle $\vartheta=51.5^{\circ}$. Based on this technique, the final angular uncertainty was found to be $\Delta \vartheta \leqslant 0.13^{\circ}$.

\section{Experimental data analysis and results}

Complete angular distributions between $20^{\circ}$ and $175^{\circ}$ were measured at energies of $E_{\alpha}=16.15$ and $19.50 \mathrm{MeV}$ in $1^{\circ}$ $\left(20^{\circ} \leqslant \vartheta \leqslant 100^{\circ}\right)$ and $2.5^{\circ}\left(100^{\circ} \leqslant \vartheta \leqslant 175^{\circ}\right)$ steps.

The statistical uncertainties varied between $0.1 \%$ (forward angles) and $4 \%$ (backward angles). The count rates $N(\vartheta)$ have been normalized to the yield of the monitor detectors $N_{\text {Mon }}\left(\vartheta=15^{\circ}\right)$ :

$$
\left(\frac{d \sigma}{d \Omega}\right)(\vartheta)=\left(\frac{d \sigma}{d \Omega}\right)_{\text {Mon }} \frac{N(\vartheta)}{N_{\mathrm{Mon}}} \frac{\Delta \Omega_{\mathrm{Mon}}}{\Delta \Omega},
$$

with $\Delta \Omega$ being the solid angles of the detectors. The relative measurement eliminates the typical uncertainties of absolute measurements, coming mainly from changes in the absolute target thickness and from the beam current integration.

The measured angular distributions are shown in Fig. 2. The lines are the result of optical model predictions using global OMPs. The measured absolute cross sections cover more than four orders of magnitude between the highest (forward angles at $E_{\alpha}=16.15 \mathrm{MeV}$ ) and the lowest cross sections (backward angle at $E_{\alpha}=19.5 \mathrm{MeV}$ ) with almost the same accuracy (4\%-5\% total uncertainty). This error is mainly caused by the uncertainty of the determination of the scattering angle in the forward region and from the statistical uncertainty in the backward region.

The origin of the above uncertainties has to be studied in further detail. The uncertainty of the scattering angle is composed of two parts. Firstly, a systematic uncertainty comes from the alignment of the angular scale and the beam direction; it affects all data points in the same direction. This uncertainty is partly compensated by the absolute normalization of the data (see below) where the data are adjusted to Rutherford scattering at forward angles. Secondly, the accuracy of setting/reading the angle leads to a statistical uncertainty, obviously different (a)

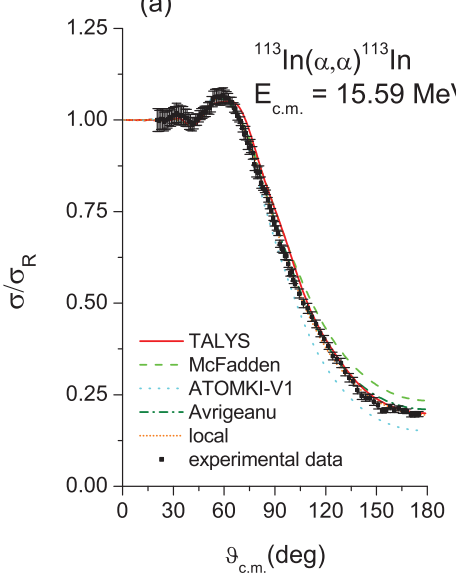

(b)

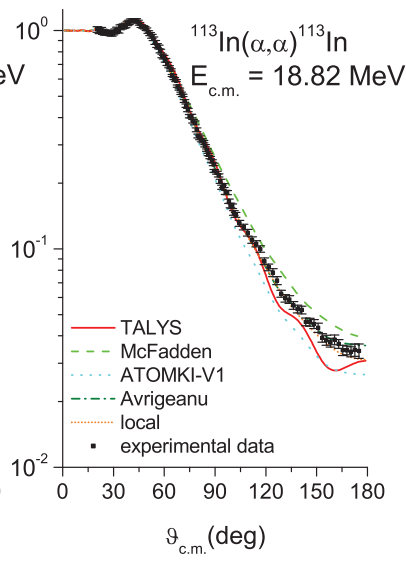

FIG. 2. (Color online) Rutherford normalized elastic scattering cross sections of ${ }^{113} \operatorname{In}(\alpha, \alpha)^{113} \operatorname{In}$ at $E_{\text {c.m. }}=15.59$ (a) and $18.82 \mathrm{MeV}$ (b) vs the angle in the center-of-mass frame. The lines correspond to predictions using different OMPs: from Watanabe [46] as used in [45] (TALYS), from [47] (McFadden), [9] (ATOMKI-V1), [48] (Avrigeanu), and using the fitted local potential described in Sec. III A (local). The contribution of the ${ }^{115} \operatorname{In}(\alpha, \alpha){ }^{115} \operatorname{In}$ elastic scattering to the presented experimental data is below $1 \%$.

for each data point. The combination of both leads to an uncertainty of the cross section which remains below $4 \%-5 \%$.

The absolute normalization is done in two steps. In the first step the absolute normalization is taken from experiment, i.e., from the integrated beam current, the solid angle of the detectors, and the thickness of the target. This procedure has a relatively large uncertainty of the order of $10 \%$, where the following partial uncertainties were taken into account: number of target atoms $(9 \%)$, current measurement $(5 \%)$, solid angle determination (5\%), and counting statistics (1\%). In the second step a "fine-tuning" of the absolute normalization is obtained by comparison to theoretical calculations at very forward angles. It is obvious that calculated cross sections from any reasonable potential practically do not deviate from the Rutherford cross section at the most forward angles of this experiment; typical deviations are below $0.5 \%$ for all potentials listed (including those potentials that do not describe details of the angular distributions at backward angles). This fine-tuning changed the first experimental normalization by only $2.5 \%$ and thus confirmed the first normalization within the given errors.

The measured ${ }^{113} \operatorname{In}(\alpha, \alpha)^{113}$ In scattering cross sections are practically not affected by the small ${ }^{115}$ In contribution in the target. According to optical model calculations, the elastic scattering cross sections of ${ }^{113}$ In and ${ }^{115}$ In deviate by less than $10 \%$ over the full angular range. This is confirmed by a new scattering experiment on ${ }^{115}$ In [49]. The small deviation of less than $10 \%$ in combination with the high ${ }^{113}$ In enrichment of $93.1 \%$ in the present work leads to an uncertainty far below $1 \%$, which can be neglected in the analysis.

\section{OPTICAL MODEL ANALYSIS}

In the following we will present a theoretical analysis of the new experimental data within the framework of the optical 
TABLE I. Parameters of the local optical potential for the ${ }^{113} \mathrm{In}+\alpha$ system.

\begin{tabular}{|c|c|c|c|c|c|c|c|c|c|c|c|}
\hline$E_{\text {c.m. }}(\mathrm{MeV})$ & \multicolumn{5}{|c|}{ Real part } & \multicolumn{5}{|c|}{ Imaginary part } & $\chi^{2} / F$ \\
\hline 18.82 & 1.198 & 1.000 & 317.6 & 5.304 & 127.0 & 1.429 & 0.459 & 78.5 & 7.154 & 758 & 0.87 \\
\hline
\end{tabular}

model. Our analysis can be extended up to $42.2 \mathrm{MeV}$ by taking into account the elastic and inelastic $\alpha$ scattering angular distributions measured between $30^{\circ}$ and $80^{\circ}$ in [50,51].

\section{A. Local alpha-nucleus optical potential}

The complex optical model potential (OMP) is given by

$$
U(r)=V_{C}(r)+V(r)+i W(r) .
$$

The real part $V(r)$ of the nuclear potential is determined by a double-folding procedure of the densities of the $\alpha$ projectile and ${ }^{113}$ In target (derived from electron scattering [52]) with an effective nucleon-nucleon interaction of the widely used DDM3Y type [53,54] (for details of the folding procedure see also $[9,55])$. The bare folding potential $V_{F}(r)$ is modified by a strength parameter $\lambda$ and a width parameter $w$ :

$$
V(r)=\lambda V_{F}(r / w) .
$$

The strength parameter $\lambda$ and the width parameter $w$ will be adjusted to the experimental ${ }^{113} \operatorname{In}(\alpha, \alpha)^{113}$ In elastic scattering angular distributions. Obviously, the width parameter $w$ should remain close to unity; otherwise, the folding potential would be questionable. The strength parameter $\lambda$ is typically around 1.1-1.4, leading to volume integrals per interacting nucleon pair of $J_{R} \approx 310-350 \mathrm{MeV} \mathrm{fm}^{3}$ [34]. (As usual, the negative signs of $J_{R}$ and $J_{I}$ are neglected in the following discussion.)

The Coulomb potential $V_{C}(r)$ is taken as usual from a homogeneously charged sphere, with the radius parameter $R_{C}$ taken from the root-mean-square ( $\mathrm{rms}$ ) radius of the bare folding potential (with $w=1$ ).

The imaginary potential $W(r)$ is parameterized by WoodsSaxon potentials of volume and surface type:

$$
W(r)=W_{V} f\left(x_{V}\right)+W_{S} \frac{d f\left(x_{S}\right)}{d x_{S}} .
$$

The $W_{i}$ are the depth parameters of the volume and surface imaginary potential, and the Woods-Saxon function $f\left(x_{i}\right)$ is given by

$$
f\left(x_{i}\right)=\left[1+\exp \left(x_{i}\right)\right]^{-1}
$$

with $x_{i}=\left(r-R_{i} A_{T}^{1 / 3}\right) / a_{i}$ and $i=V, S$ for the volume and surface part. Note that $W_{V}<0$ and $W_{S}>0$ in the chosen conventions (4) and (5) for an absorptive negative $W(r)<0$. The maximum depth of the surface imaginary potential is given by $-W_{S} / 4$ at $r=R_{S} A_{T}^{1 / 3}$.

In general, at energies far above the Coulomb barrier the volume contribution is dominating whereas at lower energies the surface component becomes more important. For the experimental energies of 15.59 and $18.82 \mathrm{MeV}$ around the Coulomb barrier it is sufficient to neglect the volume contribution $\left(W_{V}=0\right)$ and to use a pure surface imaginary potential. At both energies fits with reduced $\chi^{2} / F \lesssim 1$ were found. The parameters of these local potential fits are listed in Table I. The excellent reproduction of the experimental angular distributions is shown in Fig. 2.

The calculation of excitation functions for $\alpha$-induced reactions requires the underlying potential at all energies under study. However, the analysis of the angular distributions provides the potential only at two energies (15.59 and $18.82 \mathrm{MeV}$ ). In the following we derive a local potential for the calculation of excitation functions from the fit parameters listed in Table I. It is interesting to note that both fits in Table I have been made independently from each other. Nevertheless, the resulting parameters for the geometry of the potential are very similar. In the real part for the width parameter $w \approx 1.0$ is found with deviations of less than $1 \%$. The imaginary radius parameter $R_{S}$ varies by about $2 \%$, and the imaginary diffuseness $a_{S}$ is practically identical in both fits. Thus, the geometry of the potential is well defined by the experimental data, and for the calculation of reaction cross sections we adopt $w=1.0$ for the real geometry and the average values $R_{S}=1.44 \mathrm{fm}$ and $a_{S}=0.46 \mathrm{fm}$ for the imaginary geometry of the local potential.

The volume integral $J_{R}$ of the real part changes by about $6 \%$. But the minimum in $\chi^{2}$ is very flat at the lower energy, and fits with $\chi^{2} / F<0.6$ can be found almost for any real volume integral $J_{R}$ between 280 and $350 \mathrm{MeV} \mathrm{fm}^{3}$ (compared to the best-fit $\chi^{2} / F=0.52$ ). Because the real part of the OMP has only a small energy dependence, we adopt a volume integral of $J_{R}=320 \mathrm{MeV} \mathrm{fm}^{3}$ for the calculation of low-energy reaction data which is slightly higher than the well-defined value of 317.6 $\mathrm{MeV} \mathrm{fm}^{3}$ at $18.82 \mathrm{MeV}$, following the trend of slightly increasing $J_{R}$ towards lower energies which is also confirmed by the analysis of the $42 \mathrm{MeV}$ data (see Sec. III B).

As expected, the volume integral $J_{I}$ of the imaginary part increases with energy because of the increasing number of open reaction channels. However, it is difficult to restrict the energy dependence of $J_{I}$ from the two new experimental data points. Typical parametrizations of this energy dependence have three adjustable parameters (saturation value $J_{I, 0}$ at large energies and two parameters for the position and slope of the increase at low energies; e.g., the new global ATOMKI-V1 potential [9] uses the parametrization in Eq. (9), see Sec. III C). Therefore, in the first calculation (labeled "local1") we keep the imaginary strength $J_{I}$ at the value measured at the lower energy of $15.59 \mathrm{MeV}$. This should provide an upper limit for $J_{I}$ at even lower energies and thus an upper limit for the calculated reaction cross sections at the energies under study in [30] (see Sec. IIID). In the second calculation 
(labeled "local2") we use the energy dependence of $J_{I}$ from the recent global ATOMKI-V1 potential [9] and set the saturation value so that the results for $J_{I}$ at 15.59 and $18.82 \mathrm{MeV}$ are approximately reproduced. This leads to a minor reduction of the ATOMKI-V1 [9] saturation value from $J_{I, 0}=92.0 \mathrm{MeV} \mathrm{fm}^{3}$ [9] by $9 \%$ to $J_{I, 0}=83.7 \mathrm{MeV} \mathrm{fm}^{3}$. More details on global potentials including the ATOMKI-V1 potential [9] are given in Sec. III C and below. We note that the geometry of the imaginary potential of the ATOMKI-V1 potential [9] $\left(R_{S}=1.43 \mathrm{fm}, a_{S}=0.47 \mathrm{fm}\right)$ is practically identical to the local potential derived from ${ }^{113} \operatorname{In}(\alpha, \alpha)^{113} \operatorname{In}$ scattering in this work $\left(R_{S}=1.44 \mathrm{fm}, a_{S}=0.46 \mathrm{fm}\right)$.

In addition to the parameters of the potential, the total reaction cross section $\sigma_{\text {reac }}$ is listed in Table I. It is defined as $[56,57]$

$$
\sigma_{\text {reac }}=\frac{\pi}{k^{2}} \sum_{L}(2 L+1)\left(1-\eta_{L}^{2}\right)
$$

where $k=\sqrt{2 \mu E_{\mathrm{c} . \mathrm{m} .}} / \hbar$ is the wave number, $E_{\mathrm{c} . \mathrm{m} .}$ is the energy in the center-of-mass system, and $\eta_{L}$ and $\delta_{L}$ are the real reflexion coefficients and scattering phase shifts which are related to the complex scattering matrix by $S_{L}=\eta_{L} \exp \left(2 i \delta_{L}\right)$. The $\eta_{L}$ were derived from the local fits to the angular distributions. The resulting $\sigma_{\text {reac }}$ has typical uncertainties of about $3 \%$ at energies around and above the Coulomb barrier if the underlying angular distributions have been measured in a wide angular range with small uncertainties [41]. Larger uncertainties appear at energies significantly below the Coulomb barrier, and the lower limit for the extraction of $\sigma_{\text {reac }}$ is studied in [58]. It should be noted that a straightforward determination of $\sigma_{\text {reac }}$, using $\eta_{L}$ from fitting elastic scattering data, is only possible when compound-elastic scattering is negligible [56,57]. This is the case for the reaction studied here.

For comparison of various targets at different energies, the total reaction cross section is often presented as reduced cross section,

$$
\sigma_{\text {red }}=\sigma_{\text {reac }} /\left(A_{P}^{1 / 3}+A_{T}^{1 / 3}\right)^{2}
$$

vs the reduced energy

$$
E_{\mathrm{red}}=\left(A_{P}^{1 / 3}+A_{T}^{1 / 3}\right) E_{\mathrm{c} . \mathrm{m} .} /\left(Z_{P} Z_{T}\right) .
$$

$\sigma_{\text {red }}$ normalizes $\sigma_{\text {reac }}$ according to the geometrical size of the projectile-plus-target system, and $E_{\text {red }}$ is a comparison to the height of the Coulomb barrier. The obtained results $\sigma_{\text {red }}=$ $8.7 \mathrm{mb}(18.4 \mathrm{mb})$ at $E_{\text {red }}=1.02 \mathrm{MeV}(1.23 \mathrm{MeV})$ for the lower (higher) energy angular distribution fit perfectly in the global systematics of total reaction cross sections $[9,41]$ (see Fig. 3).

The lower limit for the extraction of $\sigma_{\text {reac }}$ from an elastic scattering angular distribution is located slightly below $E_{\text {red }}=$ $0.8 \mathrm{MeV}$ (corresponding to $E \approx 12 \mathrm{MeV}$ for ${ }^{113} \mathrm{In}$ in the present study). Finally, it should be noted that the total reaction cross section $\sigma_{\text {reac }}$ is very important for the calculation of reaction cross sections in the statistical $\mathrm{H}-\mathrm{F}$ model because the H-F model essentially distributes $\sigma_{\text {reac }}$ among the different open channels.

The energy dependence of the imaginary volume integral $J_{I}$ has also been parameterized vs the reduced energy $E_{\text {red }}$ in [9].

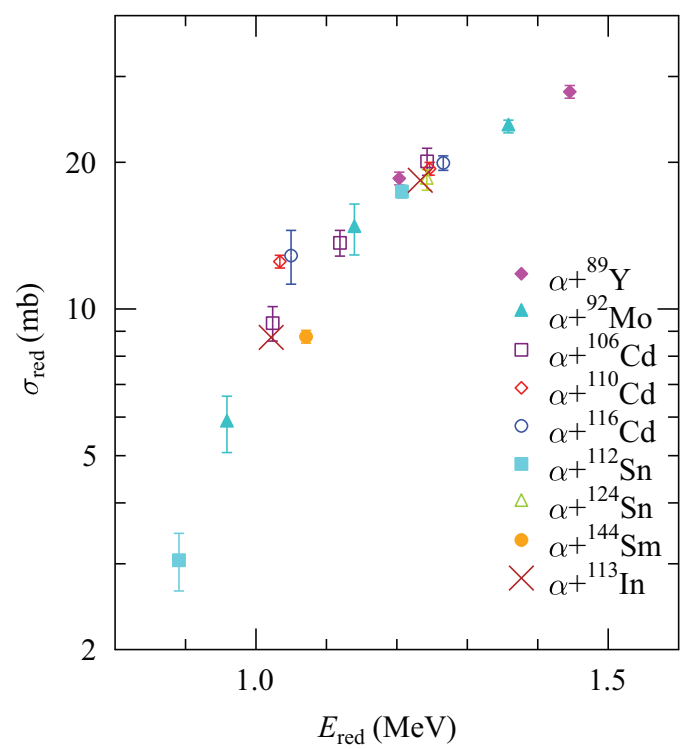

FIG. 3. (Color online) Reduced cross sections $\sigma_{\text {red }}$ vs the reduced energy $E_{\text {red }}$ for various $\alpha$-nucleus systems. The new data for ${ }^{113}$ In fit perfectly into the systematics which is taken from [9].

The new data for ${ }^{113}$ In are slightly lower than the average of the various data analyzed in [9] (see Fig. 4) but remain within the scatter of the data.

\section{B. Literature data at $42 \mathrm{MeV}$}

In addition to the study of our new low-energy scattering data, we present a detailed analysis of literature data for ${ }^{113} \operatorname{In}(\alpha, \alpha)^{113}$ In elastic scattering at the energy $E_{\text {lab }}=$ 42.2 $\mathrm{MeV}\left(E_{\text {c.m. }}=40.76 \mathrm{MeV}\right)$ [50]. This analysis nicely

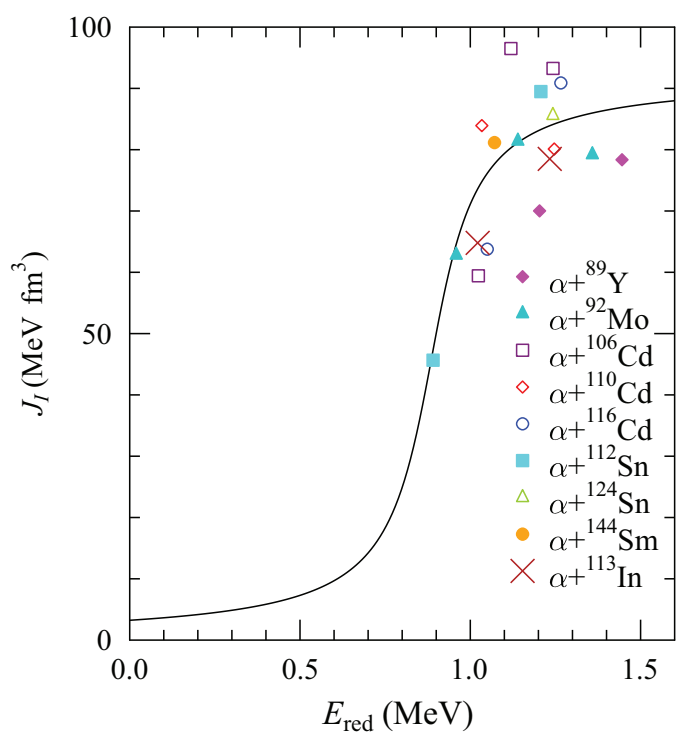

FIG. 4. (Color online) Energy dependence of the imaginary volume integral $J_{I}$ vs the reduced energy $E_{\text {red }}$ for various $\alpha$-nucleus systems. The new data for ${ }^{113}$ In are slightly lower than the average found in [9] but they remain within the scatter of the data. The line corresponds to the new ATOMKI-V1 potential [9] (see Sec. III C). 
shows that useful information on the optical potential can be extracted from old literature data; however, the information remains limited because the data in [50] do not cover the full angular range with small uncertainties.

The experimental data of [50] are shown in their Fig. 3 as "Differential cross section, $d \sigma / d \Omega$, arbitrary units" vs "Laboratory scattering angle, $\Theta_{\text {lab }}$, deg." Fortunately, the data are listed numerically in an earlier report [51], and thus digitizing of the data in Fig. 3 of [50] is not necessary. The data cover a limited angular range between about 40 and 90 degrees. The given uncertainties in [51] are statistical uncertainties only. Therefore we have added a further $5 \%$ systematic uncertainty quadratically for each data point. Additionally, the absolute cross section is relatively uncertain. It has been determined relative to elastic $\alpha$ scattering on ${ }^{115} \mathrm{In}$, and a total uncertainty of about $15 \%$ has been assigned to the absolute normalization of the ${ }^{113}$ In data [50].

A series of fits to the data of [50] has been performed using a real folding potential and imaginary Woods-Saxon potentials of volume and surface type. Reasonable fits with $\chi^{2} / F \approx 2$ are found using the numerical data of [51] with the additional $5 \%$ uncertainty. However, the resulting parameters (mainly the strengths of the real and imaginary parts) are sensitive to details of the fitting procedure (e.g., starting values). This sensitivity disappears, and the fits become very stable, as soon as the absolute normalization is also used as a fitting parameter. From the various fits we find that the data of [51] should be multiplied by a factor between 1.12 and 1.15 which is within the stated $15 \%$ uncertainty of the absolute normalization. Simultaneously, the description of the data improves to $\chi^{2} / F \approx 1.1$ for fits with a volume Woods-Saxon imaginary part and $\chi^{2} / F \approx 0.7$ for fits with a volume plus surface Woods-Saxon imaginary part. These fits are shown in Fig. 5 and compared to the experimental data (multiplied by a factor of 1.135). The parameters of the best fits with the imaginary volume-plus-surface part (imaginary volume part only) are $\lambda=1.182(1.170), w=0.998(1.004), J_{R}=$ 312.0(314.1) $\mathrm{MeV} \mathrm{fm}{ }^{3}, W_{V}=-28.3(-18.4) \mathrm{MeV}, R_{V}=$ $1.164(1.576) \mathrm{fm}, a_{V}=0.157(0.539) \mathrm{fm}, W_{S}=21.0 \mathrm{MeV}$, $R_{S}=1.513 \mathrm{fm}, a_{S}=0.627 \mathrm{fm}, J_{I}=67.1(79.3) \mathrm{MeV} \mathrm{fm}^{3}$.

Several conclusions can be drawn from this analysis.

(i) First of all, the diffraction pattern in the limited angular range of the data is sufficient to fix the radial range of the potential. This is reflected by width parameters $w$ of the real folding potential which remain very close to unity within $1 \%$ in any case (including also the fits with a fixed absolute normalization). As a consequence, the total reaction cross section is well defined by the experimental data: $1798 \leqslant$ $\sigma_{\text {reac }} \leqslant 1837 \mathrm{mb}$ for all fits. However, the strengths of the real and imaginary potentials depend on the chosen normalization of the data.

(ii) There is strong evidence that the volume integrals are about $J_{R} \approx 315 \mathrm{MeV} \mathrm{fm}{ }^{3}$ for the real part and $J_{I} \approx$ $75 \mathrm{MeV} \mathrm{fm}{ }^{3}$ for the imaginary part; these results are obtained using the revised absolute normalization. Values of up to $J_{R} \approx 350 \mathrm{MeV} \mathrm{fm}{ }^{3}$ for the real and $J_{I} \approx 120 \mathrm{MeV} \mathrm{fm}^{3}$ are obtained from fits to the original absolute normalization and thus cannot be excluded. This uncertainty could have been reduced by an extension of the experimental data to very
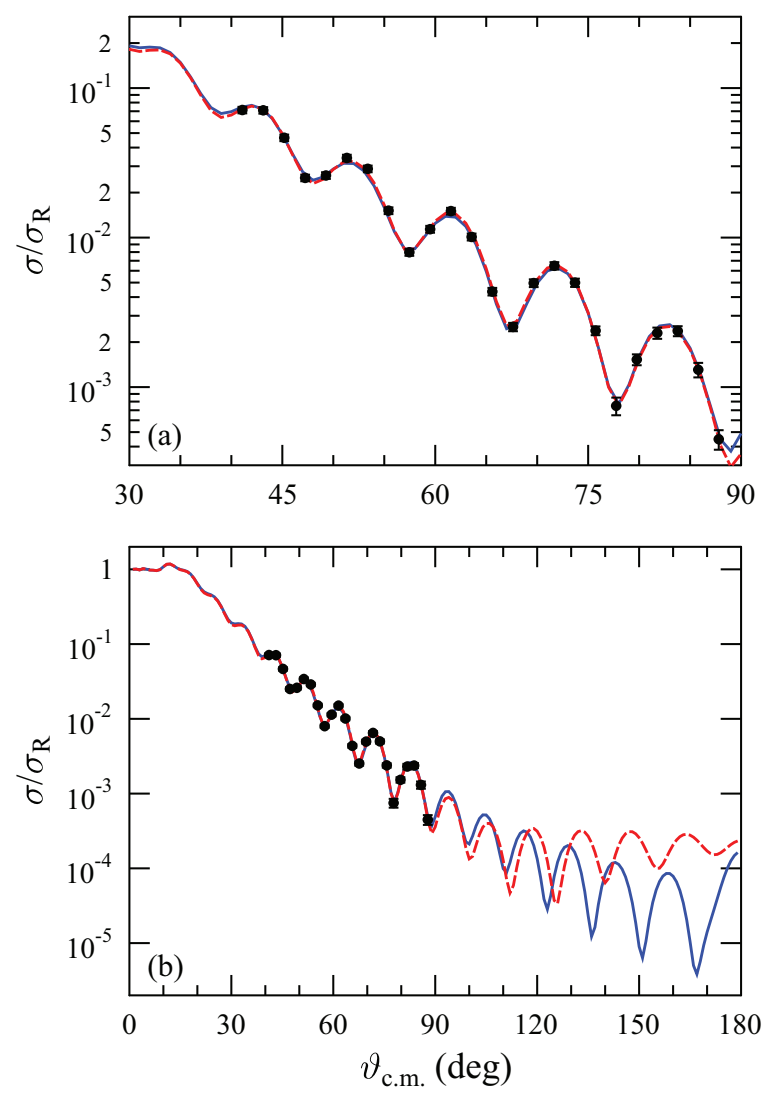

FIG. 5. (Color online) Rutherford normalized elastic scattering cross section of the ${ }^{113} \operatorname{In}(\alpha, \alpha)^{113} \mathrm{In}$ reaction at $E_{\text {lab }}=42.2 \mathrm{MeV}$ vs the angle in center-of-mass frame. The experimental data are taken from [50,51] and have been multiplied by 1.135 . The lines are fits to the data using a real folding potential and a Woods-Saxon imaginary part composed of a volume term (full blue line) and a volume-plussurface term (dashed red line). The upper part (a) shows the limited angular range where experimental data are available; the lower part (b) shows the full angular range. For further discussion see text.

forward angles (below approximately $15^{\circ}$ ) where the cross section approaches the Rutherford cross section. (Note that the most forward data point is below $10 \%$ of the Rutherford cross section and does not allow us to fix the absolute normalization in the usual way.)

(ii i) Finally, it is absolutely impossible to determine details of the shape of the imaginary potential from the available data. The shown fits in Fig. 5 with a volume Woods-Saxon imaginary part and a volume-plus-surface imaginary part are almost identical in the measured angular range (with a slightly improved $\chi^{2} / F$ for the volume plus surface imaginary part). Strong deviations between these two fits become visible only at very backward angles. Details of the imaginary potential can thus be only determined from data which cover the backward angular area.

Summarizing the above, the $42 \mathrm{MeV}$ data in [50,51] are sufficient to confirm that the folding potential (with a width parameter $w$ close to unity) is able to describe the data. Because of the weak energy dependence of the real part of the potential, this finding helps to restrict the low-energy fits. But the missing data at forward angles prevent a reliable absolute 
normalization and determination of the potential strengths of the real and imaginary parts, and the missing data at backward angles prevent the determination of the shape of the imaginary part.

\section{Global $\alpha+$ nucleus optical potentials}

In the framework of the $\gamma$ process network calculations a large number of reactions involving $\alpha$ particles ( $\alpha$-induced reactions and $\alpha$-particle emission) have to be taken into account. As the $\gamma$ process path is located in a region of unstable nuclei on the neutron-deficient side of the chart of nuclides, experimental data are practically not available to adjust potential parameters of the $\alpha+$ nucleus potential. Therefore, a global $\alpha+$ nucleus optical potential is required for the theoretical prediction of reaction cross sections involving $\alpha$ particles within the statistical H-F model. Several different parametrizations for the optical potential exist, giving very different predictions for reaction cross sections, in particular at very low energies far below the Coulomb barrier. In the following we will compare the predictions of well known or recent open access global potentials to our experimental results.

(i) The regional optical potential (ROP) of [59] was derived starting from a semimicroscopic analysis, using the double folding model [60], based on alpha-particle elastic scattering on $A \approx 100$ nuclei at energies below $32 \mathrm{MeV}$. The energy-dependent phenomenological imaginary part of this semimicroscopic optical potential takes into account also a dispersive correction to the microscopic real potential. A small revision of this ROP and especially the use of local parameter sets were able to describe the variation of the elastic scattering cross sections along the $\mathrm{Sn}$ isotopic chain [61]. A further step to include all available $\alpha$-induced reaction cross sections below the Coulomb barrier has recently been carried out [8]. First, the ROP based entirely on $\alpha$-particle elastic scattering [59] was extended to $A \sim 50-120$ nuclei and energies from $\sim 13-50 \mathrm{MeV}$. Secondly, an assessment of available $(\alpha, \gamma),(\alpha, n)$ and $(\alpha, p)$ reaction cross sections on target nuclei ranging from ${ }^{45} \mathrm{Sc}$ to ${ }^{118} \mathrm{Sn}$ at incident energies below $12 \mathrm{MeV}$ was carried out. A minor revision of this potential has been suggested very recently by Avrigeanu [48], which is used in the present study.

(ii) In recent years several elastic $\alpha$ scattering experiments have been performed at ATOMKI [35-40]. As a first step a local potential analysis with consistent standardized parameterizations of the real and imaginary parts has been performed on the high precision experimental data. Based on this study, a new few-parameter global optical potential parametrization - which gives a correct prediction for the total $\alpha$-induced reaction cross sections - has been suggested in [9]. The very few adjustable parameters of this potential avoid contingent problems which may appear in the extrapolation of many-parameter potentials for unstable nuclei with $N / Z$ ratios deviating from stable nuclei. The geometry of the energy-independent real part of the potential is determined using the folding procedure as described briefly in Sec. III A. It is characterized by the volume integral $J_{R}=371 \mathrm{MeV} \mathrm{fm}^{3}$ for nonmagic target nuclei like ${ }^{113} \mathrm{In}$. The imaginary part of the potential is described by surface Woods-Saxon potential with energy-independent radius and diffuseness parameters. The energy dependence of the imaginary part is determined using the saturation value $J_{I, 0}$, the turning point energy $E_{\text {red, } 0}$, and the slope parameter $\Gamma_{\text {red }}$ in a $J_{I}$-vs- $E_{\text {red }}$ diagram:

$$
J_{I}\left(E_{\mathrm{red}}\right)=\frac{1}{\pi} J_{I, 0} \times \arctan \left[\frac{\Gamma_{\mathrm{red}}}{2\left(E_{\mathrm{red}, 0}-E_{\mathrm{red}}\right)}\right] .
$$

We refer to this potential from [9] as ATOMKI-V1, i.e., the first version of the few-parameter ATOMKI potential.

(iii) The widely used potential by McFadden [47] is a very simple four-parameter Woods-Saxon potential with massand energy-independent parameters. Despite its simplicity it provides an excellent description of $\alpha$ scattering data and cross sections of $\alpha$-induced reactions, in particular at energies slightly above the Coulomb barrier, whereas it has a tendency to overestimate reaction cross sections at very low energies below the Coulomb barrier. This potential was used as default for the $\mathrm{H}-\mathrm{F}$ calculations of astrophysical reaction rates in the NON-SMOKER code [5,6].

(iv) Furthermore, elastic $\alpha$ scattering cross section calculations were performed using the TALYS code [45]. The optical model potential calculations within TALYS are performed with ECIS-06 [62] using a default OMP based on a simplification of the folding approach of Watanabe [46].

The results of the calculations using the various OMPs are compared to the experimental scattering data in Fig. 2. The $15.59 \mathrm{MeV}$ angular distribution is well reproduced by the default potential implemented in the TALYS code (labeled "TALYS") [45,46], it is slightly underestimated by the calculation performed using the ATOMKI-V1 potential [9], and slightly overestimated by the calculations performed using the potentials of Avrigeanu [48] and McFadden [47]. The picture is a bit different for the $18.82 \mathrm{MeV}$ angular distribution. In this case the measured data are well reproduced by the calculation using the potential of Avrigeanu [48]; again the potential of McFadden [47] overestimates the cross sections, while the calculations performed using the ATOMKI-V1 [9] and the default TALYS potential of Watanabe [46] are slightly underestimating the experimental data. For a strict comparison between the potentials the $\chi^{2}$ values and total reaction cross sections $\sigma_{\text {reac }}$ can be found in Table II.

TABLE II. $\chi^{2} / F$ and total reaction cross sections $\sigma_{\text {reac }}$ (in mb) of predictions using different global parameterizations compared with the angular distributions measured in the present work and taken from literature [50,51]. Except for those from the local fit, no parameters have been adjusted to the new experimental data.

\begin{tabular}{lcccccccc}
\hline \hline Potential & \multicolumn{2}{c}{$15.59 \mathrm{MeV}$} & & \multicolumn{2}{c}{$18.82 \mathrm{MeV}$} & & \multicolumn{2}{c}{$40.76 \mathrm{MeV}$} \\
\cline { 2 - 3 } & $\chi^{2} / F$ & $\sigma_{\text {reac }}$ & & $\chi^{2} / F$ & $\sigma_{\text {reac }}$ & & $\chi^{2} / F$ & $\sigma_{\text {reac }}$ \\
\hline Local & 0.52 & 361 & & 0.87 & 758 & & 0.75 & 1837 \\
ATOMKI-V1 [9] & 15.5 & 397 & & 22.4 & 807 & & 345 & 1811 \\
Avrigeanu [48] & 1.6 & 342 & & 1.0 & 751 & & 187 & 1742 \\
McFadden [47] & 13.0 & 326 & & 23.7 & 726 & & 191 & 1716 \\
TALYS [45] & 9.6 & 313 & & 12.0 & 703 & & 358 & 1659 \\
\hline \hline
\end{tabular}


The result of the local fit can be considered as quasiexperimental result for $\sigma_{\text {reac }}$ with an uncertainty of about $3 \%$ at energies above the Coulomb barrier and about 5\% at the lowest energy $15.59 \mathrm{MeV}$ under study. The predicted $\sigma_{\text {reac }}$ from the global potentials do not deviate by more than $10 \%-15 \%$ from the experimental result at the lowest energy of $15.59 \mathrm{MeV}$, and the agreement becomes even better with 5\%-10\% deviation at 18.82 and $40.76 \mathrm{MeV}$ for all potentials under study. An explanation for this relatively good agreement of $\sigma_{\text {reac }}$ from the various potentials is given in $[58,63]$. As expected, the ATOMKI-V1 potential [9] which is designed for low energies (with a surface imaginary part only; higher energies would require an additional volume term), shows a very poor $\chi^{2} / F$ at the highest energy. But, surprisingly, this poor $\chi^{2}$ does not affect the prediction of the total reaction cross section $\sigma_{\text {reac }}$ which is the best of all global potentials under study. The potential by Avrigeanu [48] provides excellent $\chi^{2} / F$ at the lower energies, and in particular at $18.82 \mathrm{MeV}$ a $\chi^{2} / F \approx 1.0$ leads to a $\sigma_{\text {reac }}$ very close to the experimental result.

\section{D. $\alpha$-induced reactions at sub-barrier energies on ${ }^{113}$ In}

In recent years, $\alpha$-induced reactions at sub-barrier energies on ${ }^{113}$ In have been studied using the activation technique by [30] with the aim to provide cross-section data for the modeling of the astrophysical $\gamma$ process. The cross sections of the ${ }^{113} \operatorname{In}(\alpha, \gamma){ }^{117} \mathrm{Sb}$ reaction were measured from $E_{\text {c.m. }}=$ 8.66 up to $13.64 \mathrm{MeV}$. This energy range-which lies only few hundred $\mathrm{keV}$ above the astrophysically relevant energy region located within 5.55-8.42 MeV (for plasma temperature $T=2.5 \mathrm{GK}$ ) [64] —was covered by typically $0.5 \mathrm{MeV}$ steps. Furthermore, the cross section of the ${ }^{113} \operatorname{In}(\alpha, n)^{116} \mathrm{Sb}$ reaction was measured between $E_{\text {c.m. }}=9.66$ and $13.64 \mathrm{MeV}$. Figure 6 shows the measured cross sections-presented as astrophysical $S$ factors-in comparison with the theoretical predictions calculated using the global OMP parameterizations studied in the present work. Earlier unpublished data are available at slightly higher energies above $10 \mathrm{MeV}$ for the ${ }^{113} \operatorname{In}(\alpha, n){ }^{116} \mathrm{Sb}$ and ${ }^{113} \operatorname{In}(\alpha, 2 n){ }^{115} \mathrm{Sb}$ reactions [65].

In general, the cross section of an $(\alpha, X)$ reaction in the statistical model depends on the total transmission coefficients $T_{i}$ into the open channels (note that the total transmission and average width for a particular channel are closely related; see, e.g., Eqs. (64) and (65) in [56] and [66]):

$$
\sigma(\alpha, X) \propto \frac{T_{\alpha} T_{X}}{\sum_{i} T_{i}}
$$

In many cases the sum in the denominator in Eq. (10) is dominated by the neutron channel: $\sum_{i} T_{i} \approx T_{n}$. For $\alpha$-induced reactions on ${ }^{113}$ In the reaction $Q$ values are $Q(\alpha, \gamma)=$ $+1.70 \mathrm{MeV}, Q(\alpha, n)=-8.19 \mathrm{MeV}, Q(\alpha, p)=-2.70 \mathrm{MeV}$, and $Q(\alpha, 2 n)=-16.08 \mathrm{MeV}$. Because of the high Coulomb barrier, the $(\alpha, p)$ channel remains weak and is typically two orders of magnitude below the $(\alpha, n)$ channel between 9 and $15 \mathrm{MeV}$; thus, the above condition $\sum_{i} T_{i} \approx T_{n}$ is fulfilled in this energy region.

Under these circumstances we find $\sigma(\alpha, n) \propto T_{\alpha}$ and $\sigma(\alpha, \gamma) \propto T_{\alpha} T_{\gamma} / T_{n}$ [66]. Consequently, $\sigma(\alpha, n)$ is essentially
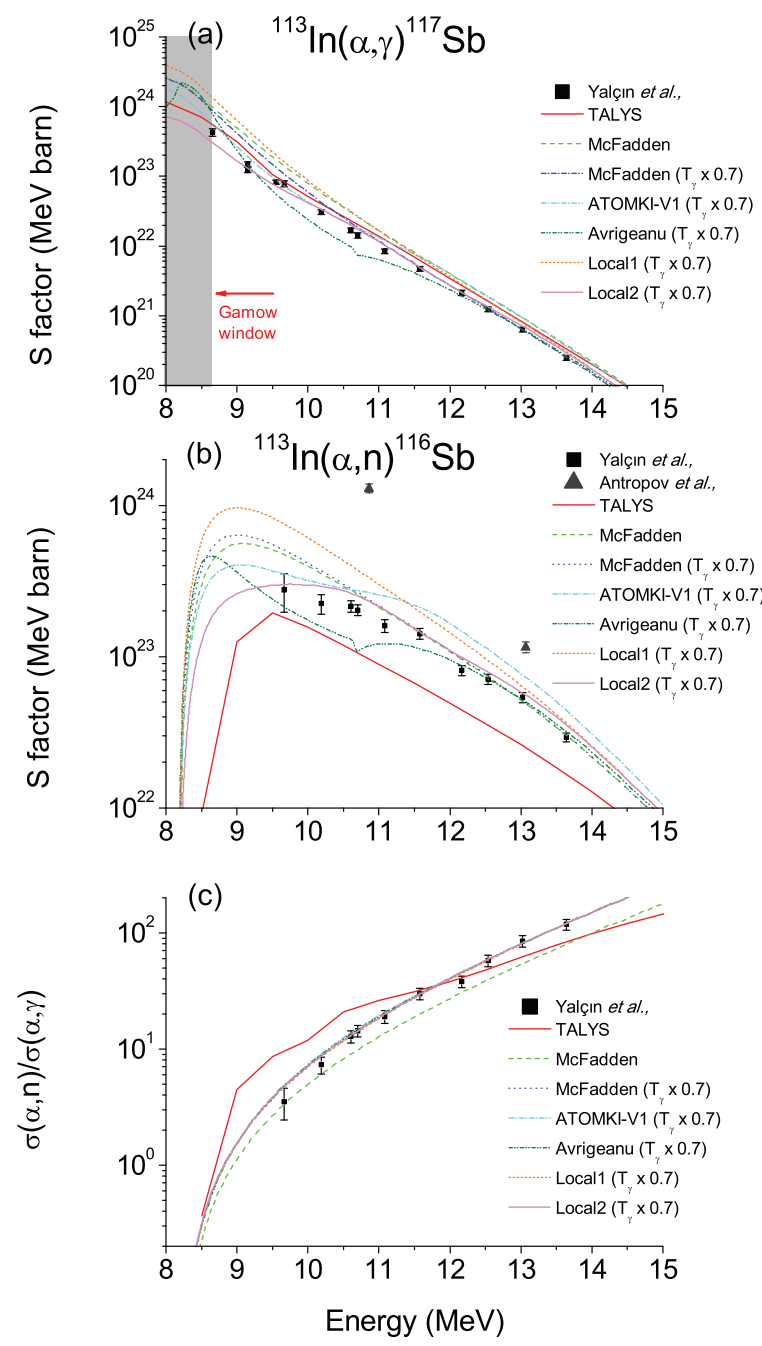

FIG. 6. (Color online) Astrophysical $S$ factor of the $\left.{ }^{113} \operatorname{In}(\alpha, \gamma)\right)^{117} \mathrm{Sb}$ and ${ }^{113} \operatorname{In}(\alpha, n){ }^{116} \mathrm{Sb}$ reactions and their ratio $S(\alpha, \mathrm{n}) / S(\alpha, \gamma)=\sigma(\alpha, \mathrm{n}) / \sigma(\alpha, \gamma)$. The lines show H-F predictions using different OMPs: obtained with TALYS [45], using the built-in version of Watanabe [46] (TALYS), and with SMARAGD using [47] (McFadden), [9] (ATOMKI-V1), [48] (Avrigeanu), and the local potentials described in Sec. III A (local1, local2). The cross sections obtained with a $\gamma$ width renormalized by a factor of 0.7 are marked by " $T_{\gamma} \times 0.7$ ". The gray area represents the upper limit of the Gamow window for $\alpha$ capture, which lies between 5.55 and $8.42 \mathrm{MeV}$ at $T=2.5$ GK [64].

defined by the $\alpha$ potential, and experimental data can be used to constrain the $\alpha$ potential. As soon as $T_{\alpha}$ is fixed, $\sigma(\alpha, \gamma)$ provides a constraint for the ratio $T_{\gamma} / T_{n}$ but it is not possible to determine $T_{\gamma}$ or $T_{n}$ individually. The following calculations have mainly been performed using the code SMARAGD [67]. Only the comparison for the reaction cross sections obtained with the potential by [46] has been made with the TALYS [45] code.

In the energy range between 12 and $14 \mathrm{MeV}$ we find excellent agreement between the experimental $(\alpha, n)$ data and most of the calculations. This clearly indicates that $T_{\alpha}$ is correctly predicted in this energy interval. However, at the same time the $(\alpha, \gamma)$ cross section is overestimated by about 
$30 \%$ in the SMARAGD calculations. This indicates a deficiency in the description of either the $\gamma$ or the neutron transmission (or both) because $\sigma(\alpha, \gamma)$ is proportional to $T_{\gamma} / T_{n}$ at these energies. This ratio depends on the nuclear input used, such as the optical potential and discrete final states for $T_{n}$ and the gamma/strength function and level density for $T_{\gamma}[56,66]$.

As the focus of the present work is the study of the $\alpha$ potential, we have simply scaled the default $T_{\gamma}$ in the SMARAGD code by a factor of 0.7 to achieve agreement with the $(\alpha, \gamma)$ data between 12 and $14 \mathrm{MeV}$. The same result can also be achieved by scaling the neutron transmission $T_{n}$ by $1 / 0.7 \approx 1.4$. As explained above, the modification of the $\gamma$ (or neutron) transmission only affects the $(\alpha, \gamma)$ cross section but not the $(\alpha, n)$ cross section. For better legibility, in Fig. 6 the unmodified result is only shown for the potentials of McFadden [47] and Watanabe [46] (TALYS). The scaling factor of 0.7 for $T_{\gamma}$ ( or 1.4 for $T_{n}$ ) can be nicely visualized by a plot of the ratio $\sigma(\alpha, n) / \sigma(\alpha, \gamma)$ which depends on the ratio $T_{n} / T_{\gamma}$ but is independent of $T_{\alpha}$ and the underlying $\alpha$-nucleus potential (see Fig. 6). Thus, the ratio $\sigma(\alpha, n) / \sigma(\alpha, \gamma)$ is an excellent measure for the further ingredients of H-F calculations beyond the $\alpha$-nucleus potential.

At energies below $12 \mathrm{MeV}$, the potential by McFadden [47] starts to overestimate the $(\alpha, n)$ and $(\alpha, \gamma)$ cross sections. This is a typical behavior for this potential, which is probably related to the missing energy dependence in particular of the imaginary part. Contrary to this, the potential by Avrigeanu [48] slightly underestimates both reaction cross sections at lower energies. The ATOMKI-V1 potential [9] shows good agreement at lower energies but slightly overestimates both reaction cross sections above $11 \mathrm{MeV}$.

As expected, the "local2" potential provides excellent agreement for both reactions over the full energy range under study whereas the "local1" potential (fixed to the $15.59 \mathrm{MeV}$ scattering data without energy dependence of the imaginary part) overestimates the reaction data at low energies. It has already been pointed out in Sec. III A that the locall potential provides an upper limit of the reaction cross sections.

Contrary to the above $\alpha$ potentials, the default TALYS potential (taken from Watanabe [46]) underestimates the $(\alpha, n)$ cross section over the full energy range and thus provides $T_{\alpha}$ which are clearly to small. Hence, the surprisingly good agreement with the $(\alpha, \gamma)$ cross sections must be considered as accidental when too small $T_{\alpha}$ are compensated by a too large $T_{\gamma} / T_{n}$ ratio. Similar to the SMARAGD calculation, $T_{\gamma} / T_{n}$ would have to be scaled, albeit by a larger factor, in TALYS and thus would yield a strongly underpredicted $(\alpha, \gamma)$ cross section.

Summarizing the above, it is shown that a locally adjusted $\alpha$ potential in combination with the energy dependence of [9] is able to reproduce the cross sections of $\alpha$-induced reactions. This finding strengthens the motivation for further scattering experiments. Contrary to the local potential, all global potentials show more or less pronounced deviations from the experimental reaction data at low energies. There is clear progress using the latest global potentials by Avrigeanu [48] or ATOMKI-V1 [9] compared to the older potentials but further improvements of these latest potentials are still required.

Finally, some remarks on the experimental $(\alpha, n)$ and $(\alpha, 2 n)$ data of [65] are in order. There are data points at
15.6 and $18.9 \mathrm{MeV}$, i.e., at almost the same energies as our new elastic scattering data. According to the EXFOR database [68], at $18.9 \mathrm{MeV}$ cross sections $\sigma(\alpha, n)=543 \pm 35 \mathrm{mb}$ and $\sigma(\alpha, 2 n)=191 \pm 17 \mathrm{mb}$ were reported. The sum of these two dominating channels is $734 \mathrm{mb}$ (at this energy the estimated cross section of the ${ }^{113} \operatorname{In}(\alpha, p)$ reaction is about $17 \mathrm{mb}$, while the ${ }^{113} \operatorname{In}(\alpha, \gamma)$ reaction cross section is below $0.5 \mathrm{mb}$ [69]) which is in good agreement with the total reaction cross section from elastic scattering $\left(\sigma_{\text {reac }}=758 \mathrm{mb}\right)$. However, at $15.6 \mathrm{MeV}$ their $\sigma(\alpha, n)=503 \pm 46 \mathrm{mb}$ significantly exceeds the total reaction cross section from elastic scattering $\left(\sigma_{\text {reac }}=\right.$ $361 \mathrm{mb}$ ) by a factor of 1.4. At even lower energies there appears an increasing discrepancy up to a factor of five to the data from [30] for the $(\alpha, n)$ reaction (at this energy the estimated cross section of the ${ }^{113} \operatorname{In}(\alpha, p)$ reaction is about $7 \mathrm{mb}$, while the ${ }^{113} \operatorname{In}(\alpha, \gamma)$ reaction cross section is below $1.5 \mathrm{mb}$ [69]). Because of the disagreement of the data of [65] with two independent subsequent experiments, we recommend disregarding these data of [65], at least at energies below $16 \mathrm{MeV}$.

\section{SUMMARY}

We have measured angular distributions of elastic ${ }^{113} \operatorname{In}(\alpha, \alpha){ }^{113}$ In scattering at $E_{\text {c.m. }}=15.59$ and $18.82 \mathrm{MeV}$. From the new experimental data and from literature data at higher energies [50] a local $\alpha$ potential for the $p$ nucleus ${ }^{113} \mathrm{In}$ has been derived. This local potential is able to reproduce the cross sections of the ${ }^{113} \operatorname{In}(\alpha, n){ }^{116} \mathrm{Sb}$ and ${ }^{113} \operatorname{In}(\alpha, \gamma){ }^{117} \mathrm{Sb}$ reactions over the whole energy range under study, and in particular at very low energies.

The derived total reaction cross sections $\sigma_{\text {reac }}$ fit nicely into the systematics of so-called reduced cross sections $[9,41]$ and are well reproduced by most global $\alpha+$ nucleus potentials within about $10 \%$. However, the global potentials cannot describe the angular distributions with the same quality as the local fit. Nevertheless, the potential by Avrigeanu [48] reaches a $\chi^{2}$ per point not far above 1.0 whereas the other global potentials show larger $\chi^{2} / F$ of $\approx 10-20$.

Contrary to the excellent reproduction of the total reaction cross sections at 15.59 and $18.82 \mathrm{MeV}$, the global potentials are not able to predict the cross section of $\alpha$-induced reactions at lower energies. This calls for further improvement of the latest global $\alpha+$ nucleus optical model potentials.

\section{ACKNOWLEDGMENTS}

This work was supported by the EUROGENESIS research program, by the Hungarian-Portuguese Intergovernmental S\&T Cooperation Programme No. TÉT_10-1-2011-0458, by the European Research Council Grant Agreement No. 203175, by OTKA (NN83261, K101328, PD104664, K108459), by the Scientific and Technical Research Council of Turkey (TUBITAK, Grant No. 109T585) and by the ENSAR/THEXO European FP7 program. G.G.K. acknowledges support from the János Bolyai program of the Hungarian Academy of Sciences. T.R. is supported by the Hungarian Academy of Sciences. C.Y. acknowledges support through The Scientific and Technical Research Council of Turkey (TUBITAK) under the program BIDEP-2219. 
[1] V. Yu. Denisov and H. Ikezoe, Phys. Rev. C 72, 064613 (2005).

[2] P. Mohr, Phys. Rev. C 73, 031301(R) (2006); 74, 069902(E) (2006).

[3] P. E. Hodgson, Contemp. Phys. 31, 295 (1990).

[4] W. Hauser and H. Feshbach, Phys. Rev. 87, 366 (1952).

[5] T. Rauscher and F.-K. Thielemann, At. Data Nucl. Data Tables 75, 1 (2000).

[6] T. Rauscher and F.-K. Thielemann, At. Data Nucl. Data Tables 79, 47 (2001).

[7] A. Palumbo, W. P. Tan, J. Görres, A. Best, M. Couder, R. Crowter, R. J. deBoer, S. Falahat, P. J. LeBlanc, H. Y. Lee, S. O’Brien, E. Strandberg, M. Wiescher, J. P. Greene, Zs. Fülöp, G. G. Kiss, E. Somorjai, N. Özkan, G. Efe, and R. T. Güray, Phys. Rev. C 85, 035808 (2012).

[8] M. Avrigeanu, A. C. Obreja, F. L. Roman, V. Avrigeanu, and W. von Oertzen, At. Data Nucl. Data Tables 95, 501 (2009).

[9] P. Mohr, G. G. Kiss, Zs. Fülöp, D. Galaviz, Gy. Gyürky, and E. Somorjai, At. Data Nucl. Data Tables 99, 651 (2013).

[10] S. E. Woosley and W. M. Howard, Astrophys. J. Suppl. 36, 285 (1978).

[11] D. L. Lambert, Astron. Astroph. Rev. 3, 201 (1992).

[12] M. Arnould and S. Goriely, Phys. Rep. 384, 1 (2003).

[13] I. Dillmann, T. Rauscher, M. Heil, F. Käppeler, W. Rapp, and F.-K. Thielemann, J. Phys. G 35, 014029 (2008).

[14] I. Dillmann, F. Käppeler, T. Rauscher, F.-K. Thielemann, R. Gallino, and S. Bisterzo, PoS NIC X, 091 (2008).

[15] Zs. Németh, F. Käppeler, C. Theis, T. Belgya, and S. W. Yates, Astrophys. J. 426, 357 (1994).

[16] T. Rauscher, N. Dauphas, I. Dillmann, C. Fröhlich, Zs. Fülöp, and Gy. Gyürky, Rep. Prog. Phys. 76, 066201 (2013).

[17] C. Travaglio, F. K. Röpke, R. Gallino, and W. Hillebrandt, Astrophys. J. 739, 93 (2011).

[18] T. Rauscher, A. Heger, R. D. Hoffman, and S. E. Woosley, Astrophys. J. 576, 323 (2002).

[19] T. Rauscher, Phys. Rev. C 73, 015804 (2006).

[20] W. Rapp, J. Görres, M. Wiescher, H. Schatz, and F. Käppeler, Astrophys. J. 653, 474 (2006).

[21] C. Nair, A. R. Junghans, M. Erhard, D. Bemmerer, R. Beyer, P. Crespo, E. Grosse, M. Fauth, K. Kosev, G. Rusev, K. D. Schilling, R. Schwengner, and A. Wagner, J. Phys. G 35, 014036 (2008).

[22] P. Mohr, Zs. Fülöp, and H. Utsunomiya, Eur. Phys. J. A 32, 357 (2007).

[23] G. G. Kiss, T. Rauscher, Gy. Gyürky, A. Simon, Zs. Fülöp, and E. Somorjai, Phys. Rev. Lett. 101, 191101 (2008).

[24] T. Rauscher, G. G. Kiss, Gy. Gyürky, A. Simon, Zs. Fülöp, and E. Somorjai, Phys. Rev. C 80, 035801 (2009).

[25] Zs. Fülöp, Á. Z. Kiss, E. Somorjai, C. E. Rolfs, H. P. Trautvetter, T. Rauscher, and H. Oberhummer, Z. Phys. A 355, 203 (1996).

[26] W. Rapp, M. Heil, D. Hentschel, F. Käppeler, R. Reifarth, H. J. Brede, H. Klein, and T. Rauscher, Phys. Rev. C 66, 015803 (2002).

[27] Gy. Gyürky, G. G. Kiss, Z. Elekes, Zs. Fülöp, E. Somorjai, A. Palumbo, J. Görres, H. Y. Lee, W. Rapp, M. Wiescher, N. Özkan, R. T. Güray, G. Efe, and T. Rauscher, Phys. Rev. C 74, 025805 (2006).

[28] N. Özkan, G. Efe, R. T. Güray, A. Palumbo, J. Görres, H. Y. Lee, L. O. Lamm, W. Rapp, E. Stech, M. Wiescher, Gy. Gyürky, Zs. Fülöp, and E. Somorjai, Phys. Rev. C 75, 025801 (2007).
[29] I. Cata-Danil, D. Filipescu, M. Ivascu, D. Bucurescu, N. V. Zamfir, T. Glodariu, L. Stroe, G. Cata-Danil, D. G. Ghita, C. Mihai, G. Suliman, and T. Sava, Phys. Rev. C 78, 035803 (2008).

[30] C. Yalçın, R. T. Güray, N. Özkan, S. Kutlu, Gy. Gyürky, J. Farkas, G. G. Kiss, Zs. Fülöp, A. Simon, E. Somorjai, and T. Rauscher, Phys. Rev. C 79, 065801 (2009).

[31] E. Somorjai, Zs. Fülöp, Á. Z. Kiss, C. E. Rolfs, H. P. Trautvetter, U. Greife, M. Junker, S. Goriely, M. Arnould, M. Rayet, T. Rauscher, and H. Oberhummer, Astron. Astrophys. 333, 1112 (1998).

[32] Gy. Gyürky, Z. Elekes, J. Farkas, Zs. Fülöp, Z. Halász, G. G. Kiss, E. Somorjai, T. Szücs, R. T. Güray, N. Özkan, C. Yalçın and T. Rauscher, J. Phys. G 37, 115201 (2010).

[33] G. G. Kiss, T. Rauscher, T. Szücs, Zs. Kertész, Zs. Fülöp, Gy. Gyürky, C. Fröhlich, J. Farkas, Z. Elekes, and E. Somorjai, Phys. Lett. B. 695, 419 (2011).

[34] U. Atzrott, P. Mohr, H. Abele, C. Hillenmayer, and G. Staudt, Phys. Rev. C 53, 1336 (1996).

[35] P. Mohr, T. Rauscher, H. Oberhummer, Z. Máté, Zs. Fülöp, E. Somorjai, M. Jaeger, and G. Staudt, Phys. Rev. C 55, 1523 (1997).

[36] Zs. Fülöp, Gy. Gyürky, Z. Máté, E. Somorjai, L. Zolnai, D. Galaviz, M. Babilon, P. Mohr, A. Zilges, T. Rauscher, H. Oberhummer, and G. Staudt, Phys. Rev. C 64, 065805 (2001).

[37] G. G. Kiss, Zs. Fülöp, Gy. Gyürky, Z. Máté, E. Somorjai, D. Galaviz, A. Kretschmer, K. Sonnabend, and A. Zilges, Eur. Phys. J. 27, 197 (2006).

[38] D. Galaviz, Zs. Fülöp, Gy. Gyürky, Z. Máté, P. Mohr, T. Rauscher, E. Somorjai, and A. Zilges, Phys. Rev. C 71, 065802 (2005).

[39] G. G. Kiss, P. Mohr, Zs. Fülöp, D. Galaviz, Gy. Gyürky, Z. Elekes, E. Somorjai, A. Kretschmer, K. Sonnabend, A. Zilges, and M. Avrigeanu, Phys. Rev. C 80, 045807 (2009).

[40] G. G. Kiss, P. Mohr, Zs. Fülöp, Gy. Gyürky, Z. Elekes, J. Farkas, E. Somorjai, C. Yalçın, D. Galaviz, R. T. Güray, N. Özkan, and J. Görres, Phys. Rev. C 83, 065807 (2011).

[41] P. Mohr, D. Galaviz, Zs. Fülöp, Gy. Gyürky, G. G. Kiss and E. Somorjai, Phys. Rev. C 82, 047601 (2010).

[42] G. G. Kiss, D. Galaviz, Gy. Gyürky, Z. Elekes, Zs. Fülöp, E. Somorjai, K. Sonnabend, A. Zilges, P. Mohr, J. Görres, M. Wiescher, N. Özkan, T. Güray, C. Yalçın, and M. Avrigeanu, in Origin of Matter and Evolution of Galaxies, The 10th International Symposium, Sapporo, Japan, 2007, edited by T. Suda, T. Nozawa, A. Ohnishi, K. Kato, M. Y. Fujimoto, T. Kajino, and S. Kubono, AIP Conf. Proc. No. 1016 (AIP, New York, 2008), p. 221.

[43] http://www.ortec-online.com

[44] J. Blachot, Nucl. Data Sheets 83, 647 (1998).

[45] A. Koning, S. Hilaire, and M. Duijvestijn, code TALYS version 1.4, available online: http://www.talys.eu/

[46] S. Watanabe, Nucl. Phys. 8, 484 (1958).

[47] L. McFadden and G. R. Satchler, Nucl. Phys. 84, 177 (1966).

[48] M. Avrigeanu and V. Avrigeanu, Phys. Rev. C 82, 014606 (2010).

[49] G. G. Kiss et al., J. Phys. Conf. Ser. (to be published).

[50] W. M. Stewart, N. Baron, and R. F. Leonard, Phys. Rev. 171, 1316 (1968).

[51] W. M. Stewart, N. Baron, and R. F. Leonard, National Aeronautics and Space Administration Technical Note D-4222, 1967 (unpublished). 
[52] H. de Vries, C. W. de Jager, and C. de Vries, At. Data Nucl. Data Tables 36, 495 (1987).

[53] G. R. Satchler and W. G. Love, Phys. Rep. 55, 183 (1979).

[54] A. M. Kobos, B. A. Brown, R. Lindsay, and G. R. Satchler, Nucl. Phys. A 425, 205 (1984).

[55] H. Abele and G. Staudt, Phys. Rev. C 47, 742 (1993).

[56] T. Rauscher, Int. J. Mod. Phys. E 20, 1071 (2011).

[57] E. Gadioli and P. E. Hodgson, Pre-Equilibrium Nuclear Reactions (Clarendon, Oxford, 1992).

[58] P. Mohr, Phys. Rev. C 87, 035802 (2013).

[59] M. Avrigeanu, W. von Oertzen, A. J. M. Plompen, and V. Avrigeanu, Nucl. Phys. A 723, 104 (2003); M. Avrigeanu, W. von Oertzen, and V. Avrigeanu, ibid. 764, 246 (2006).

[60] D. T. Khoa, W. von Oertzen, and H. G. Bohlen, Phys. Rev. C 49 1652 (1994).
[61] M. Avrigeanu and V. Avrigeanu, Phys. Rev. C 73, 038801 (2006).

[62] http://www.oecd-nea.org/tools/abstract/detail/nea-0850

[63] P. Mohr, Phys. Rev. C 84, 055803 (2011).

[64] T. Rauscher, Phys. Rev. C 81, 045807 (2010).

[65] A. E. Antropov, V. G. Batij, V. P. Gusev, A. A. Kolozhvari, E. A. Skakun, and A. V. Smirnov, in Proceedings of the 40th Annual Conference in Nuclear Spectroscopy and Nuclear Structure, Leningrad, 1990 (unpublished), p. 343.

[66] T. Rauscher, Astrophys. J. Suppl. 201, 26 (2012).

[67] T. Rauscher, computer code SMARAGD, version $0.9 .1 \mathrm{~s}$ (2012).

[68] EXFOR database, http://www-nds.iaea.org/exfor/exfor.htm, version September 21, 2012.

[69] http://nucastro.org/nonsmoker.html 